\title{
Effects of massage on physiological restoration, perceived recovery, and repeated sports performance
}

\author{
Brian Hemmings, Marcus Smith, Jan Graydon, Rosemary Dyson
}

\begin{abstract}
Background-Despite massage being widely used by athletes, little scientific evidence exists to confirm the efficacy of massage for promoting both physiological and psychological recovery after exercise and massage effects on performance.

Aim-To investigate the effect of massage on perceived recovery and blood lactate removal, and also to examine massage effects on repeated boxing performance.

Methods-Eight amateur boxers completed two performances on a boxing ergometer on two occasions in a counterbalanced design. Boxers initially completed performance 1 , after which they received a massage or passive rest intervention. Each boxer then gave perceived recovery ratings before completing a second performance, which was a repeated simulation of the first. Heart rates and blood lactate and glucose levels were also assessed before, during, and after all performances.
\end{abstract}

Results-A repeated measures analysis of variance showed no significant group differences for either performance, although a main effect was found showing a decrement in punching force from performance 1 to performance $2(p<0.05)$. A Wilcoxon matched pairs test showed that the massage intervention significantly increased perceptions of recovery $(p<0.01)$ compared with the passive rest intervention. A doubly multivariate multiple analysis of variance showed no differences in blood lactate or glucose following massage or passive rest interventions, although the blood lactate concentration after the second performance was significantly higher following massage $(\mathbf{p}<0.05)$.

Conclusions-These findings provide some support for the psychological benefits of massage, but raise questions about the benefit of massage for physiological restoration and repeated sports performance.

(Br F Sports Med 2000;34:109-115)

Keywords: massage; lactate; psychological recovery; physiological recovery; performance

Massage has been a therapeutic modality in most cultures since early civilisation and has had a long tradition of use in sport. ${ }^{12}$ Frequent claims made in the sports literature for the benefits of massage include improved stretching of tendons and connective tissue ${ }^{3}$ and relief of muscle tension and spasm. ${ }^{4}$ Massage is also commonly assumed to enhance muscle recovery from intense exercise, principally because it speeds up muscle blood flow. ${ }^{6}$ However, studies to date on blood flow are contradictory. A previous review ${ }^{7}$ pointed out that reports on limb blood flow vary from no effect of massage to as much as a $50 \%$ increase. For instance, positive effects have been reported showing that massage promotes acceleration of muscle and venous blood flow, increases blood volume, and reduces muscle tightness. ${ }^{89}$ In contrast, more recent research ${ }^{10}{ }^{11}$ showed no effect of massage on blood flow irrespective of the type of massage stroke or the muscle mass being treated.

There is a widely held belief that accumulated lactate is at least partially responsible for retarding recovery from fatigue. ${ }^{12}{ }^{13}$ As it has been proposed that massage could increase blood flow to the muscles being massaged, and blood flow is suggested to be an important factor in the removal of lactate after exercise through enhanced oxidation and diffusion out of the muscles, ${ }^{14-16}$ studies have sought to determine whether massage has any effect on lactate removal after an exercise bout.

Research findings have only partially supported a positive effect of massage on lactate removal. For instance, although blood lactate levels after exercise were shown to be significantly lower following a massage compared with a passive rest condition, a warm down intervention was seen to promote the most efficient lactate removal. ${ }^{17}$ In addition, other researchers have shown superior lactate disappearance in active recovery compared with massage and rest conditions after exercise. ${ }^{18-20}$ It is widely acknowledged that lactate removal is enhanced during active recovery ${ }^{21}$ and these findings further suggest that active warm down strategies may be more efficient than massage at removing lactate during the recovery phase. However, there is still some doubt about whether massage may be more effective than rest in removing blood lactate and hence enable more efficient physiological recovery for athletes between training sessions or performances.

Furthermore, previous authors have recognised that psychological regeneration after intense training or sports performance must be examined in conjunction with physiological restoration. $^{22} 23$ Few empirical studies have focused on the psychological response to
Accepted for publication 28 October 1999 
massage, and the evidence that does exist is equivocal. ${ }^{24}$ There is at present little published research that has investigated whether massage can help counteract any negative psychological effects with respect to fatigue and perceived recovery between performances. ${ }^{26}$

There are also frequent anecdotal claims in the sports literature that massage can enhance performance, ${ }^{3}$ yet the evidence that currently exists on the effects of massage on performance is equivocal. ${ }^{27-33}$ However, many sports also involve repeated performance, where the ability to recover quickly from the effects of fatigue is important and massage is often recommended as a way to improve recovery. ${ }^{27}$

One study that examined the role of massage when repeated performance was required found no effect of massage on physiological markers or on performance in elite cyclists. ${ }^{34}$ Performance times in a four day stage race were no different whether a daily massage after each stage or a no massage condition was used. Nevertheless, it must be recognised that between the daily performances there was a substantial period of rest as well as the massage treatment. This factor may have been important, as the cyclists may have had sufficient time to recover without the need for massage intervention. In contrast, other repeated sport performance conditions exist in which stricter time demands apply and massage could have an effect. Amateur boxing is one such example; in domestic competition, victorious competitors may have as little as a one hour rest period before boxing new opponents. Moreover, boxing has traditionally included massage as part of bout preparation and it is commonplace to see boxers receiving massage before competition.

Indeed, in a study with less time between performances, $45 \%$ improvements in subsequent exercise performances were found following a 20 minute massage recovery period when compared with a passive recovery condition. ${ }^{20}$ The finding that cardiorespiratory and blood variables showed no differences between conditions prompted the authors to suggest a psychological pathway through which massage could positively influence recovery and subsequent performance. ${ }^{20}$

In summary, previous research on the effect of massage on blood lactate removal after intense exercise has produced equivocal results, ${ }^{7}$ and despite numerous anecdotal testimonies to its efficacy, little scientific research exists on the effects of massage on psychological parameters or on repeated sport performance. The potential for massage to influence psychological, physiological, and performance aspects has been highlighted, and if it is considered that recovery from exercise constitutes an interplay between psychological and physiological factors, ${ }^{22}{ }^{35}$ then it would seem that a concurrent analysis is necessary to evaluate any relation. Therefore the aim of this interdisciplinary study was to investigate the effect of massage on blood lactate removal after amateur boxing performance and to examine massage effects on perceived recovery and repeated sports performance. It was hypoth- esised that massage would lead to increased psychological regeneration, but it was unclear if massage would lead to greater lactate removal or improved amateur boxing performance.

\section{Methods}

PARTICIPANTS

Eight male amateur boxers aged 24.9 (3.8) years, height $1.8(0.07) \mathrm{m}$ (mean (SD)), volunteered to participate in the study and signed informed consent forms. All had participated in the sport for a minimum period of two years. The study received approval from the University College Chichester ethics committee.

\section{MEASURES}

Heart rates and blood lactate and glucose concentrations

During boxing performances, heart rate was recorded and stored to memory for subsequent analysis (Polar Sports Tester, Bodycare, Kempele, Finland). Fingerprick $(50 \mu \mathrm{l})$ capillary blood samples were drawn to determine blood lactate and glucose concentrations (2300 StatPlus; YSI, Ohio, USA). The lactate and glucose automated analyser was calibrated before each experimental trial using 2.5, 5.0, 7.5, and 15 $\mathrm{mM}$ lactate and 5.0 and $10.0 \mathrm{mM}$ glucose standards respectively.

\section{Perceived recovery}

Boxers were requested to give responses to four items on a 0 (not at all) to 7 (very much so) linear rating scale designed to explore perceptions of recovery (total scores ranged between 0 and 28). Numbers 1 to 6 on this scale were undefined and were used to delineate degrees of perceived recovery between the two ends of the scale. The items were "refreshed", "recharged", "rested", and "recovered". The scale has good internal consistency $(\alpha=0.86)$, and face validity of the included items was established through scrutiny by a panel of four British Association of Sport and Exercise Sciences accredited sport psychologists. ${ }^{37} \mathrm{~A}$ similar rating scale has been used to assess perceptions of sluggishness and heaviness in athletes. $^{38}$

\section{Boxing performance}

The design, construction, and calibration of the boxing ergometer system has been described in detail. ${ }^{39}$ Briefly, the ergometer uses matched 9366 force sensors (Kistler, Winterthur, Switzerland) which provide the identification of a pathway of force production for each type of punch due to three dimensional sensitivity. Previous results have indicated that the ergometer is a valid and reliable instrument, and is capable of discriminating between elite, intermediate, and novice boxers in a range of punches. ${ }^{39}$ In this study, punching force measured in Newtons was recorded. Mean peak force was determined per punch in each round, providing a measure of boxing performance. As the punching protocol was straight lead and rear hand punches either singularly or in two and three punch combinations, the force data collected in the $\mathrm{Fz}$ (anterior-posterior) plane was selected for 
analysis. The Fx (medio-lateral) and Fy (vertical) planes are considered more applicable to force measurement in hook and uppercut punches. $^{39}$

PROCEDURE

All participants were habituated to punching the boxing ergometer in the two week period before the beginning of the study. Participants maintained a normal diet throughout the experimental period and refrained from intensive training. They entered the laboratory having refrained from physical activity over the previous 48 hours. Each participant's mass (Avery scales, Avery, Birmingham, UK; calibrated $\pm 100 \mathrm{~g}$ ) was determined before he completed performance 1 , a simulated boxing bout which consisted of five two minute rounds, interspersed with one minute seated recovery periods. This comprised eighty straight punches per round, performed in a prescribed sequence from an audio cue. A ten minute self selected warm up comprising stretching, jogging, and hitting hand held coaching pads (Bryan, London, UK) was conducted before the bout simulation. Crepe hand bandages (2.5 $\mathrm{m}$ length, $0.05 \mathrm{~m}$ width) were worn by each participant underneath competition gloves (284 g; Top Ten, Berlin, Germany).

Immediately after performance 1, participants undertook one of two 20 minute interventions. The interventions were massage and no massage. Massage was applied by the same qualified sports massage therapist and consisted of a standard 20 minute routine encompassing the major muscle groups of the legs (eight minutes), back (two minutes), and shoulders and arms (10 minutes). As available literature on duration of massage sets no established time, the 20 minute routine was chosen to replicate recent studies on massage and exercise recovery. ${ }^{17} 182040$ A stopwatch was worn by the therapist to ensure standardisation of time. The order in which the massage techniques were administered to body parts was consistent throughout treatments. The selected routine was designed to target the main muscles used in boxing. ${ }^{41}$ Effleurage (30 strokes/min) and petrissage (50-60 strokes/ min) massage techniques were used. Effleurage massage consisted of rhythmic pressure strokes along the longitudinal axis of each muscle group in a distal to proximal fashion. Petrissage consisted of kneading and squeezing motions over the muscle mass. Participants received treatment while lying in the prone position followed by the supine position.

In the passive rest intervention, participants lay resting on mats. After the intervention, participants had a further 35 minute rest period, whereupon they completed another self selected warm up, and a second performance, which was a repeated simulation of performance 1 . Therefore there was a one hour period between the two boxing performances, the minimum period for domestic amateur boxing competition.

Participants rated themselves on perceived recovery after each intervention. Peak heart rate was recorded after each round in both per-

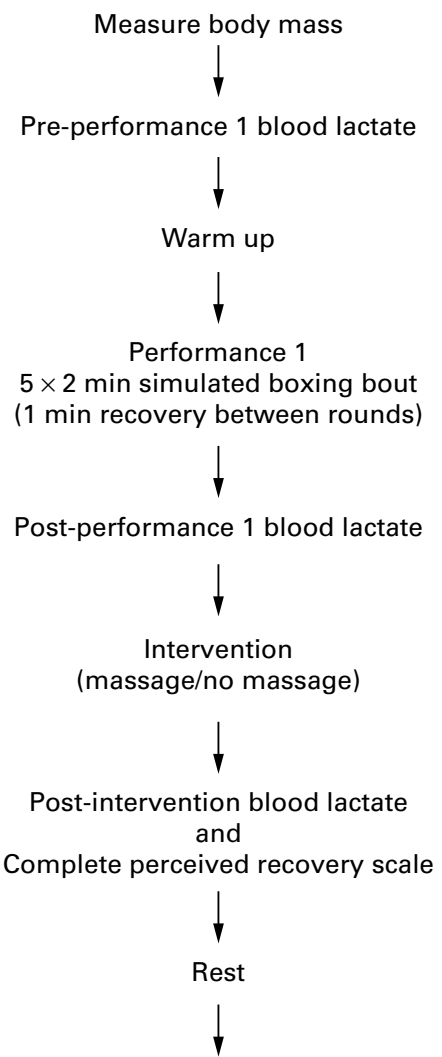

Pre-performance 2 blood lactate

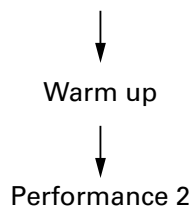

$5 \times 2$ min simulated boxing bout

(1 min recovery between rounds)

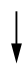

Post-performance 2 blood lactate

Figure 1 Experimental design.

formances, and blood for lactate and glucose determination was withdrawn immediately before and after performance 1, immediately after the intervention, and immediately before and after performance 2 . Figure 1 shows the complete experimental design.

Each participant performed the two trials in a counterbalanced design, separated by a seven day recovery period. Four boxers completed the experimental procedures receiving massage as the first intervention and completed the procedures again seven days later, receiving the rest intervention. The remaining four boxers completed the same procedures receiving interventions in reverse order.

\section{STATISTICAL ANALYSIS}

A doubly multivariate multiple analysis of variance with follow up univariate analysis was used to investigate differences in blood lactate and glucose levels between conditions. For heart rates and punching force, repeated measures analysis of variance was used, with Tukey's post hoc test to locate differences between the 
Table 1 Peak heart rate (beats/min) after rounds in performance 1 and 2 for massage and passive rest interventions

\begin{tabular}{lll}
\hline & Massage & Passive rest \\
\hline $\begin{array}{l}\text { Performance 1 } \\
\text { Round 1 }\end{array}$ & $169(6)$ & $173(6)$ \\
Round 2 & $178(4)$ & $178(5)$ \\
Round 3 & $181(4)$ & $180(5)$ \\
Round 4 & $183(4)$ & $181(5)$ \\
Round 5 & $184(5)$ & $182(5)$ \\
Performance 2 & & \\
Round 1 & $175(5)$ & $172(6)$ \\
Round 2 & $180(5)$ & $179(6)$ \\
Round 3 & $183(5)$ & $181(5)$ \\
Round 4 & $185(5)$ & $183(6)$ \\
Round 5 & $187(5)$ & $185(5)$ \\
\hline
\end{tabular}

Values are mean (SEM)

Table 2 Blood glucose concentration ( $m M$ ) in massage and passive rest interventions

\begin{tabular}{lll}
\hline Sampling point & Massage & Passive rest \\
\hline Before performance 1 & $4.57(0.25)$ & $4.72(0.19)$ \\
After performance 1 & $5.70(0.36)$ & $5.19(0.28)$ \\
After intervention & $4.70(0.27)$ & $5.06(0.33)$ \\
Before performance 2 & $3.99(0.22)$ & $4.43(0.30)$ \\
After performance 2 & $5.31(0.23)$ & $5.06(0.21)$ \\
\hline
\end{tabular}

Values are mean (SEM).

means. A Wilcoxon matched pairs test was employed for perceived recovery analysis. A probability level of 0.05 was used to ascertain significance. Finally, a Pearson's product correlation analysis was used to assess the relation between perceived recovery and blood lactate.

\section{Results}

BODY MASS

A paired $t$ test (two tailed) showed no significant differences between interventions for body mass before performance 1 (massage, 78.17 (8.16) kg; passive rest, $78.27(9.11) \mathrm{kg} ; t_{7}$ $=-0.43, \mathrm{p}>0.05)$.

HEART RATES AND BLOOD LACTATE AND GLUCOSE CONCENTRATIONS

Heart rates (table 1) were examined using a 2 (intervention) $\times 2$ (performance) $\times 5$ (round) repeated measures analysis of variance, with all independent variables as repeated measures. This showed a significant main effect for round (round $1<$ rounds $2-5, \quad F_{4,28}=44.35$, $\mathrm{p}<0.0001$ ), but no main effects for intervention $\left(F_{1,7}=0.4, \mathrm{p}>0.05\right)$ or performance $\left(F_{1,7}=\right.$ $4.12, \mathrm{p}>0.05)$. No significant two way or three way interactions were found $(\mathrm{p}>0.05)$.

Blood lactate (fig 2) and glucose (table 2) were analysed by a doubly multivariate multiple analysis of variance. This showed a significant time $\times$ intervention interaction, $\lambda=0.41$ $\left(F_{8,54}=3.72, \mathrm{p}<0.005\right)$. Follow up univariate analysis showed that both lactate $\left(F_{4,28}=5.68\right.$, $\mathrm{p}<0.005)$ and glucose $\left(F_{4,28}=3.72, \mathrm{p}<0.05\right)$ contributed to the results. Post hoc Tukey analysis showed that blood lactate levels were higher for the massage intervention than for the passive rest intervention after performance 2 (fig 2). All other sampling points showed no differences between interventions. Post hoc Tukey analysis of blood glucose means disclosed no differences between the massage and passive rest interventions at each of the sampling points.

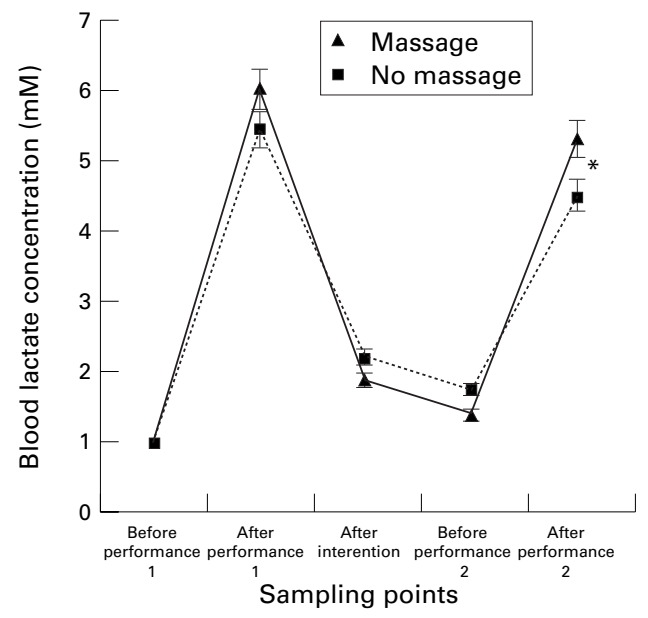

Figure 2 Mean (SEM) blood lactate concentration (mM) in massage and passive rest interventions. ${ }_{\star}$ Significant difference between interventions after performance $2(p<0.05)$.

Table 3 Punching force (N) in performance 1 and 2 for massage and passive rest interventions

\begin{tabular}{lll}
\hline Performance and round & Massage & Passive rest \\
\hline Performance 1 round 1 & $1206(69)$ & $1220(77)$ \\
Performance 1 round 2 & $1283(68)$ & $1267(89)$ \\
Performance 1 round 3 & $1300(73)$ & $1230(90)$ \\
Performance 1 round 4 & $1275(71)$ & $1226(85)$ \\
Performance 1 round 5 & $1263(72)$ & $1239(82)$ \\
Performance 2 round 1 & $1196(72)$ & $1179(86)$ \\
Performance 2 round 2 & $1246(68)$ & $1209(84)$ \\
Performance 2 round 3 & $1239(60)$ & $1207(84)$ \\
Performance 2 round 4 & $1234(66)$ & $1220(89)$ \\
Performance 2 round 5 & $1288(68)$ & $1254(84)$ \\
\hline
\end{tabular}

Values are mean (SEM).

PERCEIVED RECOVERY

Analysis of perceived recovery with a Wilcoxon matched pairs test (one tailed) showed a significant difference between interventions (massage, 19.0 (3.8); passive rest, 12.1 (4.0); $Z$ $=-2.38, \mathrm{p}<0.01)$.

BOXING PERFORMANCE

Punching force (table 3 ) was analysed with a 2 (intervention) $\times 2$ (performance) $\times 5$ (round) repeated measures analysis of variance, with all independent variables as repeated measures factors. Significant main effects were found for performance (performance $1>$ performance 2, $F_{1,7}=7.31, \mathrm{p}<0.05$ ) and round (round $1<$ rounds $\left.2-5, F_{4,28}=6.38, \mathrm{p}<0.001\right)$. No significant main effects were found for intervention $\left(F_{1,7}=0.31, \mathrm{p}>0.05\right)$. Analysis of interaction effects showed that only the performance $\times$ round interaction was significant $\left(F_{4,28}=\right.$ $4.59, \mathrm{p}<0.01)$. Post hoc Tukey analyses showed lower punching force values in performance 2 round 1 than in performance 1 rounds 2 to 5 . Finally, the two and three way interactions involving intervention were all non-significant $(\mathrm{p}>0.05)$.

\section{RELATION BETWEEN BLOOD LACTATE AND}

PERCEIVED RECOVERY

To assess the relation between psychological and physiological variables, perceptions of recovery and the blood lactate response after performance 2 were subjected to correlational analysis (fig 3). This showed a significant positive relation $(r=0.50, \mathrm{p}<0.05)$. 


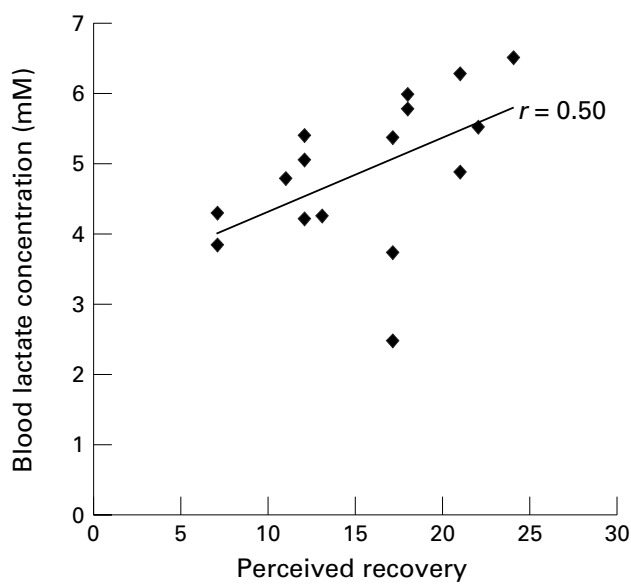

Figure 3 Scatterplot showing relation between perceived recovery and blood lactate concentration after performance $2(r=0.50, p<0.05)$.

\section{Discussion}

The major finding of this study is that massage did not affect repeated sports performance. This supports the work of Drews et al, ${ }^{34}$ who found no change in repeated cycling performance after massage treatment. Boxing has different physiological demands from cycling, and hence these findings of no effect on performance may permit generalisation to other sports with similar demands. The results showed a mean reduction in punching force in performance 2 in both conditions, hence in this case massage was unable to prevent a decrement in repeated performance. However, the finding of no difference between experimental conditions in performance 2 also supports the conclusion that massage does not have detrimental effects on performance. ${ }^{28}$

Nevertheless, there was evidence of individual variability which appears to have contributed to a finding of no difference in punching force between conditions in the second performance. Although it is evident that there is a trend in group means towards greater punching force in performance 2 after massage, it should be noted that a similar trend of higher mean scores in the massage condition existed in performance 1 before any intervention. Moreover, the observed mean differences in punching force can be argued to be so small $(14-70 \mathrm{~N})$ that they are of little clinical relevance or practical value to the boxer.

Much of the physiological basis of massage use in sport is argued to be anecdotal evidence ${ }^{7}$ hence this study also provides scientific evidence of the lack of influence of massage on physiological recovery from exercise. This research into the blood lactate response to massage supports other studies that have documented no differences between massage and passive rest conditions. ${ }^{18-20}$ Therefore only one previous study found that massage led to superior lactate removal over passive rest. ${ }^{17}$ The reasons for this isolated finding are not clear, although it is possible that the high resting blood lactate levels recorded in that study may have influenced the results. It is widely acknowledged that blood lactate is removed more quickly during active recovery because blood flow remains elevated through the active muscles, which enhances both lactate diffusion out of the muscles and its oxidation. ${ }^{21}$ Thus the physiological basis of more rapid lactate removal stimulated by massage is increased blood flow. ${ }^{6}$ As the exact effects of massage on blood flow are still being debated, ${ }^{8-11}$ blood flow was not measured in this study. Nevertheless, the present finding of no effect of massage on blood lactate would suggest that either blood flow was not affected or that it was not increased sufficiently to enhance lactate removal.

The discovery that perceptions of recovery showed significant changes after massage gives some scientific support for the use of massage as a recovery strategy. A number of authors have argued that the benefits of massage are more psychological than physiological, ${ }^{7132}$ and the finding of no physiological effects in this study supports such claims. Few explanations have been suggested with regard to potential pathways for massage to enhance positive affect. One study found a $16 \%$ increase in plasma endorphin concentration after massage, and it was suggested that the release of endorphins may explain the sensation of wellbeing following massage.$^{42}$ In addition, it has been proposed that massage could promote a feeling of wellbeing through decreased arousal levels. ${ }^{43}$

The process of monitoring for perceived recovery after each intervention also provided a new dimension to the study of psychological recovery after massage. Previous authors have suggested the use of new monitoring tools to evaluate different aspects of psychological recovery in athletes. ${ }^{44}$ Suitably, the four item section on perceptions of recovery was designed with these comments in mind, and also a similar 0-7 rating scale has been used to measure perceptions of muscle fatigue/soreness and tiredness in athletes. ${ }^{38}$ The results obtained in this study point to powerful effects of massage on perceived recovery; however, these findings should be taken as preliminary evidence and further research is needed to document the use of this scale.

The need for interdisciplinary research to explain aspects of recovery has been highlighted previously, ${ }^{45}$ and the combination of psychological and physiological measures used in this investigation may shed more light on the significantly higher blood lactate levels after performance 2 in the massage condition, a finding that could not be explained by differences in the availability of glucose as an energy source between conditions. Here it could be argued that the psychological benefits gained through massage may have resulted in boxers expending greater physical effort in the second performance. Correlational analysis partially supports such a proposition, with perceived recovery being significantly related to blood lactate concentration after performance $2(r=0.50)$. Nevertheless, submaximal heart rates were not elevated in the second performance after the massage intervention, and moreover there was no increase in boxing performance.

The finding that punching force was significantly lower in round 1 than in rounds $2-5$ 
could be explained by an insufficient warm up by the boxers in this study. Heart rate data showed lower values in round 1 than in later rounds, which also indicates that the routine chosen by boxers may not have adequately prepared them for the performance ahead. In practical terms, this information may be useful to boxers and coaches alike, as it shows that the force of the punches could be compromised in the early part of a contest if the warm up is in any way neglected.

In conclusion, this study has documented some evidence of psychological regeneration following massage. However, the efficacy of massage for promoting blood lactate removal after a boxing performance has been questioned, as has the use of massage as a performance enhancement modality. Therefore the use of massage for the latter purposes when repeated performances are required in amateur boxing in one day would not seem to be justified. Finally, it has been suggested that some relation may exist between the proposed psychological benefits of massage in the recovery phase and aspects of repeated sport performance.

Contributors: B H initiated the study and formulated the hypotheses, designed the protocol, and was responsible for data collection, analysis, and writing of the paper. He also acts as guarantor. M S was responsible for the construction of the boxing ergometer, assisted in the study design as well as participating in data collection and analysis. J G discussed issues in the study and the interpretation of findings. R D provided technical expertise and support with the ergometer and associated software.

1 Callaghan MJ. The role of massage in the management of the athlete: a review. Br f Sports Med 1993;27:28-33.

2 Goats GC. Massage: the scientific basis of an ancient art. Part 1. The techniques. Br f Sports Med 1994;28:149-52.

3 Samples P. Does sports massage have a role in sports medicine? Physician and Sportsmedicine 1987;15:177-83.

4 Ryan A. The neglected art of massage. Physician and Sportsmedicine 1980:8:25.

5 Stamford B. Massage for athletes. Physician and Sportsmedicine 1985;13:176.

6 Ylinen J, Cash M. Sports massage. London: Stanley Paul, 1988 .

7 Cafarelli E, Flint F. The role of massage in preparation for and recovery from exercise. Physiotherapy in Sport 1993;16: $17-20$

8 Dubrovsky V. Changes in muscle and venous blood flow after massage. Soviet Sports Review 1983;18:134-5.

9 Dubrovsky V. The effect of massage on athletes' cardiorespiratory systems. Soviet Sports Review 1990;25:36-8.

10 Shoemaker J, Tiidus P, Mader R. Failure of manual massage to alter limb blood flow: measures by Doppler ultrasound. Med Sci Sports Exerc 1997;29:610-14.

11 Tiidus P, Shoemaker J. Effleurage massage, muscle blood flow and long-term post-exercise strength recovery. Int $\mathcal{F}$ Sports Med 1995;16:478-83.

12 MacClaren D, Gibson H, Parry-Billings M, et al. A review of the metabolic and physiological factors in fatigue. In: Pan-
dolf $\mathrm{K}$, ed. Exercise and sport sciences reviews. Baltimore: dolf K, ed. Exercise and sport
Williams and Wilkins, 1989.

13 Maughan R, Gleeson M, Greenhaff P. Biochemistry of exercise and training. Oxford: Oxford University Press, 1997.

14 Bonen A, Belcastro A. Lactic acid removal rates during controlled and uncontrolled recovery exercise. $\mathcal{F}$ Appl Physiol 1975;39:932-6.

15 Dodd S, Powers S, Callender T, et al. Blood lactate disappearance at various intensities of recovery exercise. $f$ Appl Physiol 1984;57:1462-5.

16 Gaesser G, Brooks G. Metabolic bases of post exercise oxygen consumption. Med Sci Sport Exerc 1984;16:29-43.
17 Bale P, James H. Massage, warm-down and rest as recuperative measures after short-term intense exercise. Physiotherapy in Sport 1991;13:4-7.

18 Dolgener F, Morien A.The effect of massage on lactate disappearance. Fournal of Strength Conditioning Research 1993; 7:159-62.

19 Gupta S, Goswami A, Sadhukhan K, et al. Comparative study of lactate removal in short term massage of extremities, active recovery and a passive recovery period after supramaximal exercise sessions. Int f Sports Med 1996;17: 106-10.

20 Zelikovski A, Kaye C, Fink G, et al. The effects of the modified intermittent sequential pneumatic device (MISPD) on exercise performance following an exhaustive exercise exercise performance following an
bout. Br f Sports Med 1993;27:255-9.

21 Wilmore J, Costill D. Physiology of sport and exercise. Champaign, IL: Human Kinetics, 1994.

22 Calder A. Recovery: restoration and regeneration as essential components within training programmes. Excel 1990;6:15-19.

23 Javorek I. Methods to enhance recovery and reduce overtraining. Natural Strength Conditioning Association fournal 1987;9:43-7.

24 Weinberg R, Jackson A, Kolodny K. The relationship of massage and exercise to mood enhancement. The Sport Psychologist 1988;2:202-11.

25 Drews T, Kreider R, Drinkard B, et al. Effects of post-event massage therapy on psychological profiles of exertion, feeling and mood during a four day ultraendurance cycling event. Med Sci Sport Exerc 1991;23:91.

26 Kreider R, Hill D, Horton G, et al. Effects of carbohydrate supplementation during intense training on dietary patsupplementation during intense training on dietary pat-
terns, psychological status, and performance. Int $\mathcal{f}$ Sport terns, psychological $1995 ; 5: 125-35$.

27 Ask N, Oxelbeck U, Lundeberg T, et al. The influence of massage on quadriceps function after exhaustive exercise. Med Sci Sports Exerc 1987;19:53.

28 Balke B, Anthony J, Wyatt F. The effects of massage reatment on exercise fatigue. Clin Sports Med 1989;1:18996.

29 Rinder A, Sutherland C. An investigation of the effects of massage on quadriceps performance after exercise fatigue. Complementary Therapies Nursing Midwifery 1995;1:99102.

30 Cafarelli E, Carolan B, Sim J, et al. Vibratory massage and short-term recovery from muscular fatigue. Int $\mathcal{F}$ Sports Med 1990;11:474-8.

31 Wiktorsson-Moller M, Ekstrand J, Gillquist J. Effects of warming up, massage and stretching on range of motion and muscle strength in the lower extremity. Am 7 Sports Med 1983:11:249-52.

32 Harmer P. The effect of pre-performance massage on stride frequency in sprinters. Athletic Training 1991;26:55-9.

33 Boone T, Cooper R, Thompson W. A physiological evaluation of the sports massage. Athletic Training 1991;26:51-4.

34 Drews T, Kreider R, Drinkard, B, et al. Effects of post-event massage therapy on repeated ultra-endurance cycling. Int $\mathcal{F}$ Sports Med 1990;11:407.

35 Fry R, Morton A, Keast D. Overtraining in athletes. Sports Med 1991;12:32-65.

36 Wylie J. The general significance of recovery or rest as a component of sports training. Fournal of Psycho-Social Aspects 1981 Apr:57-66.

37 Hemmings B. Psychological and immunological effects of training and massage in amateur boxing. Doctoral thesis: University of Southampton, 1998.

38 Morgan W, Costill D, Flynn M, et al. Mood disturbance following increased training in swimmers. Med Sci Sports Exerc 1988;20:408-14.

39 Smith M. Sport specific ergometry and the physiology of amateur boxing. Doctoral thesis: University of Southampton, 1998.

40 Viitasalo J, Niemela K, Kaappola R et al. Warm underwater water-jet massage improves recovery from intense physical exercise. Eur F Appl Physiol 1995;71:431-8.

41 Hickey $\mathrm{K}$. The amateur boxing association coaching manual. London: Kaye and Ward, 1980.

42 Kaada B, Torsteinbo O. Vasoactive intestinal polypeptides in connective tissue massage. Gen Pharmacol 1987;18:37984.

43 Longworth J. Psychophysiological effects of slow stroke back massage in normotensive females. Advances in Nursing Science 1982 Jul:44-61.

44 Hooper S, MacKinnon L. Monitoring overtraining in athletes. Sports Med 1995;20,321-7.

45 Burwitz L, Moore P, Wilkinson D. Future directions for performance related research in the sport sciences:an interdisciplinary approach. London: Sports Council, 1992.

Take home message

Massage seems to be a popular technique to aid recovery for athletes. Its use for physiological recovery and improved performance appears to be in doubt; however, its potential to bring psychological benefits should not be overlooked. 


\section{Commentary}

Dr Hemmings and his colleagues have made innovative use of a Kistler force plate to provide functionally relevant data on boxing performance. Previous massage studies have used cyclists and runners, probably because performance was easier to evaluate and possibly because of the place of massage in cycling folklore. Therefore this analysis on a different set of athletes and performance parameters is welcome.

There are a couple of observations. Firstly, punching force in performance 2 was decreased by $2 \%$ whether or not the boxer received massage. This highlights the fact that whereas there are physiological strategies available to help an athlete recover from the last performance and prepare for the next performance, massage does not appear to be one of them. Secondly, although massage had a minimal effect on physiological and performance parameters, the boxers themselves felt better after the massage intervention. This indicates that of the physiological, psychological, and performance outcome measures, 20 minutes of effleurage massage was found only to affect the second of these. As the authors concede, these findings should be regarded as preliminary but suggests that there is some relation between psychological and performance parameters which merits further investigation.

The removal of lactate has long been proposed by masseurs as a rationale for their techniques. This study has shown massage to be no different from passive recovery in removal of blood lactate. This finding, along with similar results of other research in runners, ${ }^{1}$ should finally lay this particular ghost to rest.

MICHAEL J CALLAGHAN

1 Dolgener FA, Morien A. The effect of massage on lactate disappearance. Fournal of Strength and Conditioning Research 1993;7:159-62. 